\title{
Red blood cells in retinal vascular disorders
}

Rupesh Agrawal National Healthcare Group Eye Institute, Tan Tock Seng Hospital, Singapore, Institute of Ophthalmology, University College London, London, Department of Mechanical Engineering, University College London, London, School of Material Science and Engineering, Nanyang Technological Universtiy, Singapore

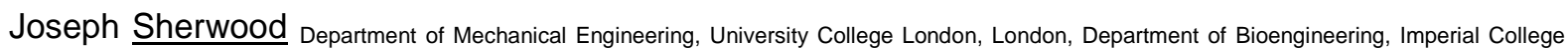
London, London

Jay Chhablani L. V. Prasad Eye Institute, Hyderabad, India

Ashutosh Ricchariya L. V. Prasad Eye Institute, Hyderabad, India

Sangho Kim Department of Biomedical Engineering, National University of Singapore, Singapore

Philip H Jones Department of Physics and Astronomy, University College London, London

Stavroula Balabani Department of Mechanical Engineering, University College London, London

David Shima Institute of Ophthalmology, University College London, London

Keywords: Blood flow; RBC; Hemorheological disorders; Rheology; RBC deformability; retinal vascular disorders

Financial Support: Nil

Conflict of interest: Nil

Contact information:

Adj Asst Prof Rupesh Agrawal,

National healthcare Group Eye Institute,

Tan Tock Seng Hospital,

School of Material Science and Engineering, Nanyang Technological University,

Singapore

Email: rupesh_agrawal@ttsh.com.sg 


\section{Table of Contents}

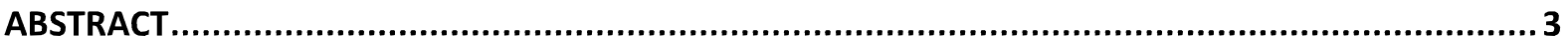

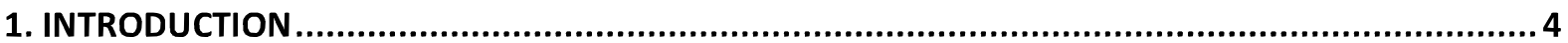

2. THE VASCULAR SYSTEM AND RETINAL MICROVASCULATURE.........................................5

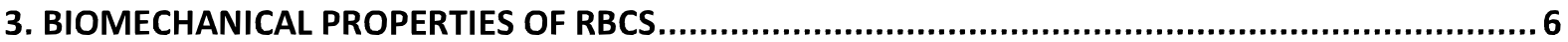

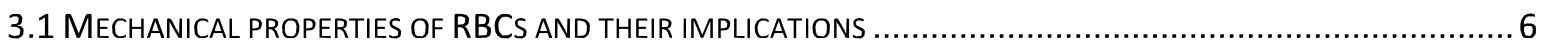

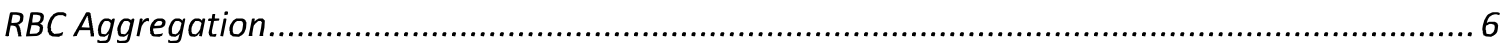

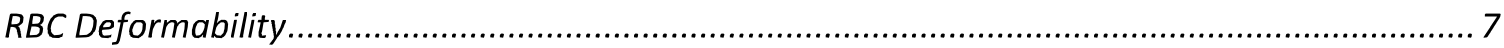

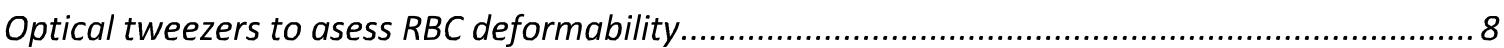

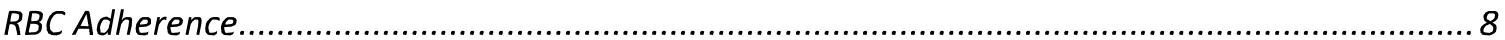

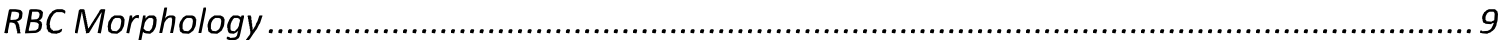

$3 D$ reconstruction of $R B C$ using defocussing microscopy .............................................. 9

3.2 BIOCHEMICAL PROPERTIES OF RBC AND THEIR ROLE IN HAEMODYNAMICS .................................... 10

4. OCULAR IMPLICATIONS OF ALTERED MICROHAEMODYNAMICS - AN OPHTHALMOLOGIST

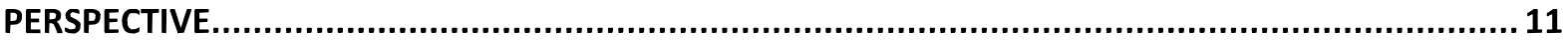

5. FUNDAMENTAL CONCEPTS IN HAEMODYNAMICS: A BIOFLUID DYNAMICISTS PERSPECTIVE ..... 13

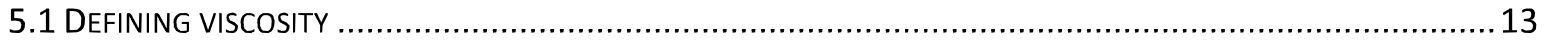

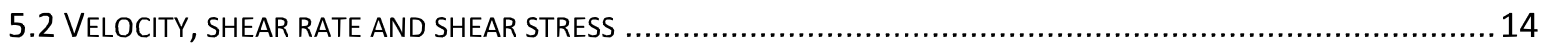

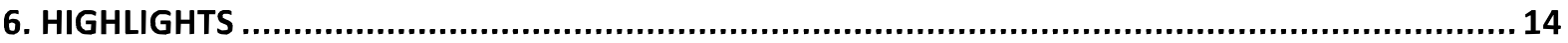

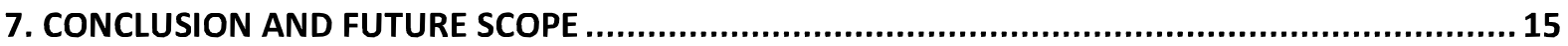

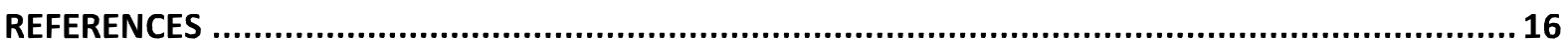

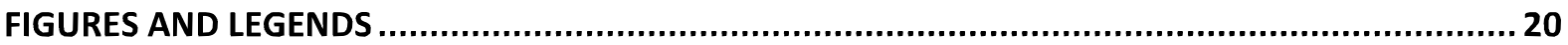




\begin{abstract}
Microvascular circulation plays a vital role in regulating physiological functions, such as vascular resistance, and maintaining organ health. Pathologies such as hypertension, diabetes, or hematologic diseases affect the microcirculation posing a significant risk to human health. The retinal vasculature provides a unique window for non-invasive visualisation of the human circulation in the vivo and retinal vascular image analysis has been established to predict the development of both clinical and subclinical cardiovascular, metabolic, renal and retinal disease in epidemiologic studies.

Blood viscosity which was otherwise thought to play a negligible role in determining blood flow based on Poiseuille's law till 1970s has now been shown to play equally if not more important role in controlling microcirculation and quantifying blood flow. Understanding the hemodynamics/rheology of the microcirculation and its changes in diseased states remains a challenging task; this is due to the particulate nature of blood, the mechanical properties of the cells (such as deformability and aggregability) and the complex architecture of the microvasculature.

In our review, we have tried to postulate possible role of red blood cell (RBC) biomechanical properties and laid down future framework for research related to hemorrheological aspects of blood in patients with retinal vascular disorders.
\end{abstract}




\section{Introduction}

There is still a significant knowledge gap in our understanding of the role of red blood cells (RBCs) as pro-oxidants or antioxidants in the pathophysiology of vascular disorders and particularly those of the retina. Due to the role of RBCs in disease pathogenesis, characterisation of the properties of a patient's RBCs could provide useful clinical information as a prognostic and biological marker to follow up vascular and inflammatory disorders.[1;2;3] In the early $20^{\text {th }}$ century, Robin Fåhraeus described the flow properties of the blood and reported, for the first time, altered suspension stability characteristics and fluidity of blood in the process of disease. [4] He explained humoral (RBC) pathology concepts on the basis of 'modern' scientific approaches and introduced the Erythrocyte Sedimentation Rate (ESR), a test which is widely used across all the sub-specialities of medicine. Unfortunately, it was not until late $20^{\text {th }}$ century, that the medical fraternity began to accept the importance of the mechanics of blood flow and there was a significant increase in research effort looking at altered haemodynamics in almost all vascular disorders.[5] A recent review by Pretorius and Kell provided an overall view of biophysical properties of RBCs, correlations with fibrin and ferritin profile and its implications in pathorheology of vascular disorders.[6]. However, the focus on the flow of RBCs was limited. The finite size of RBCs plays a significant role in modulating blood flow in microvessels with diameter less than several hundred microns. The apparent viscosity of the blood is defined by RBC dynamics and their behaviour in different vessel diameters and velocities, and their local and bulk concentrations. The biomechanics of RBCs in diverging bifurcations play a major role in distribution of blood flows and also RBC flux within the complex microvascular network.[7] In their comprehesive review, Popel et al. described the substantial heterogenity in the distribution of RBCs within the microvascular network, affecting the velocity and concentration in vascular segments of similar size. The heterogeneous distribution of blood cells is a hallmark feature of the microcirculation, influencing its exchange function and oxygen concentration in the tissues.[8]

The microcirculation refers to the smallest vessels in the vasculature, comprising vessels of size less than 100um: the arterioles, capillaries and venules.[8] Any changes in the microcirculation as a result of altered architecture or flow properties of the blood can impact the perfusion and oxygenation of the tissues, resulting in ischaemia and necrosis. Flow resistance is also determined by numerous rheological parameters including RBC aggregation, RBC adherence to endothelial cells, bulk and local haematocrit and RBC deformability. $[9 ; 10 ; 11 ; 12 ; 13 ; 14 ; 15]$ These factors may vary in individual vessel segments of the network. The inability of a microcirculatory network, including the retinal-choroidal microvasculature, to respond to haemodynamic and neurogenic signals has important clinical implications, often associated with endothelial dysfunction and pathogenesis of vascular disease.[16; 17]

The microvascular network embedded within the retinal and choroidal tissues is physiologically essential in providing the principal transport mechanism for exchange (oxygen, nutrients and metabolic waste) between the blood stream and surrounding ocular tissues, in order to maintain homeostasis and retinal-choroidal function. Retinal blood vessels are endowed with a continuous single layer of smooth muscle cells, which enables their dynamic adaptation in diameter to changes in both local blood flow properties and signals from the autonomous nervous system. However, the adaptability of the choroidal vasculature is not well understood, and the structure 
of choroidal vessels is different from that of retinal microvasculature and is quite fenestrated.

There are numerous studies on the retinal microvascular geometrical calibre assessment and its role in prediction of ischaemic events, but there are very limited studies on ocular flow dynamics and its impact on peripheral tissue perfusion and oxygenation. This article will review the possible role of haemodynamic alterations in ocular vascular disorders (Figure 1) in the context of current understanding and research in blood flow dynamics (Figure 1) and also set out a framework for future research on haemodynamics in ocular microcirculation.

\section{The vascular system and retinal microvasculature}

Blood is a complex two phase fluid, made up of plasma and formed elements. The formed elements consist of RBCs or erythrocytes, white blood cells (WBCs or leukocytes) and platelets. RBCs are the predominant cell with a single $\mu$ l of human blood containing 5 million RBCs ( 99\% of cellular the component) compared to 150 400 thousand platelets and 5-10 thousand WBCs. The primary role of RBCs is the transportation and delivery of oxygen to the peripheral tissues, including the retina. However, their mechanical and flow properties are responsible for the complex fluid dynamics which occur in microvessels. As a corollary, altered biomechanical properties of RBCs can result in impaired oxygen and nutrient supply to peripheral tissues [18], as well as altered haemodynamics. However, the complexities of microvascular blood flow are all too often simplified to analogies with Poiseuille flow. WBCs, in addition to their primary role in inflammation and immune system, also play a part in modulating blood flow, [19] while platelets are involved in thrombus formation.[20]

In between the arteries and veins lies microvasculature where the delivery of oxygen to the cells takes place and $80 \%$ of the pressure drop in the circulation occurs. [8] The microvasculature is made up of arterioles, capillaries and venules. Arterioles can be considered to be vessels smaller than $\sim 100 \mu \mathrm{m}[8]$ and are important in regulating the pressure in the capillaries by modulating their diameter and $50-60 \%$ of the pressure drop in the vasculature occurs in the arterioles.[21] Arterioles sequentially bifurcate and eventually feed into capillaries. Capillary diameters are generally similar to or less than the major diameter of the RBCs, i.e. $8 \mu \mathrm{m}$. [8; 22]

Retinal circulation is one such end circulation system, wherein capillaries terminate within the retinal and choroidal tissue before getting drained by the venular system. Approximately $15 \%$ of the total vascular pressure drop occurs in the capillary bed.[21] Retinal capillaries essentially consist of an endothelial monolayer covering the basal lamina, allowing diffusion of water, small solutes and lipid soluble materials in physiological states. However, the diffusion of RBCs and plasma proteins is inhibited.[8] An interesting phenomenon, termed vasomotion, occurs in the retinal capillary circulation, wherein the contraction and dilatation of the precapillary sphincter takes place approximately every 5 seconds, resulting in oscillatory distribution of flow in the capillary bed.[8] Venules collect the deoxygenated blood from the capillary bed and converge to form larger venules which range from 8 to $100 \mu \mathrm{m} .[23]$ 
Due to the large size of arteries and veins relative to the RBCs, it is often considered reasonable to assume that the blood behaves as a Newtonian fluid. The validity of this assumption requires further analysis, and is likely to be increasingly inaccurate in a pathological flow environment. In the microvasculature, however, the finite size and varying concentration of the RBCs has an increasingly large effect, and the Newtonian assumption is not applicable.

\section{Biomechanical properties of RBCs}

RBCs are involved in the control of circulatory processes by numerous mechanisms. Abnormal RBCs may play a role in retinal vascular disorders and stasis retinopathy by their mechanical properties. Our proposed hypothesis is that a complex cascade of cellular events involving RBC aggregation and increased blood viscosity, may lead to stagnation of the blood flow in retinal vessels and occlusion of the retinal capillary network. This will subsequently activate the endothelial cells and platelets by modulating shear stresses and attenuating the flow rate in the microvasculature and also promote WBC margination and adhesion to vessel wall endothelium. This hypothesis has not however been investigated in great detail in the either animal model or human eyes. With evolving concepts about microfluidics and also significant advances in the field of ocular imaging, we should be able to investigate this cellular mechanics in ocular pathology involving retinal vasculature.

\subsection{Mechanical properties of RBCs and their implications}

The mechanical properties of RBCs are the most important determinants of blood rheology. Healthy RBCs undergo deformation and align with the blood flow while exhibiting tank-treading, tumbling, or swinging motions, depending on the flow regime. Recently, Dupire and coworkers have shown that when RBCs flip during flow, their orientation is determined by the shear rate. With normal shear rate, there is rolling motion of RBCs and also under moderate stress, the biconcave shape of $\mathrm{RBC}$ remains stable.[24] Altered morphology of RBC, altered RBC membrane surface area to cell volume and cytoplasmic viscosity affect RBC deformability. In addition to their deformability, RBCs undergo a reversible aggregation process, forming multicellular stacked aggregates termed 'rouleaux', or clumped aggregates and may also adhere to the endothelial cells, both of which affecting the blood viscosity[25; 26; 27] Overall altered RBC mechanical properties can have significant implications in pathorheology of ocular vascular disorders which remain to be investigated.

\section{RBC Aggregation}

RBCs have a propensity to reversibly bond to one another in the presence of plasma proteins or long chain polymers such as Dextran 500. RBC aggregation is often considered to only have an influence in venules, due to the low mean shear rates in these vessels. However, even for the 'Poiseuille' case of a parabolic velocity profile, the shear rate in the channel centre is very small. The blunting and skewing of the velocity profiles of blood flow will often enhance these regions of low shear. RBC aggregation is the major determinant of blood viscosity under low local shear 
conditions due to the increased effective particle size of aggregates which results in significant flow disturbances [28] and pronounced blood viscosity. Both RBC aggregation and deformability can affect blood flow in smaller blood vessels and in the microcirculation.[25; 26; 29; 30] Interestingly, RBC aggregation is present in athletic, but not in sedentary species, implying a positive, beneficial role of aggregation. However, to what extent aggregation has a positive beneficial role and what is the limit beyond which aggregation can result in pathological changes is not yet understood.[31]

RBC aggregation in human is traditionally assessed by the Erythrocyte Sedimentation Rate (ESR) which is a widely used clinical laboratory test in retinal vascular pathologies and ocular inflammatory diseases. However, being only a measurement of the rate of RBC sedimentation, it is only indirectly a measurement of RBC aggregation in the patient. Thus, even though the test is widely followed, it does not provide information about RBC factors that might play a role in the pathophysiology. As a result, treatment strategies are not generally directed to treat some of these intrinsic factors of RBCs, which can play a major role in the pathophysiology of disease. Based on principles of photometry, [32]; or image processing applied on sheared RBC suspensions [33] new automated methods have been developed to quantify and investigate RBC aggregation but such methods are not yet in clinical use, as it is still not clear what the role of RBC aggregation is.

\section{RBC Deformability}

RBCs are highly specialised cells shaped as biconcave disks of about $8 \mu \mathrm{m}$ in diameter and $2 \mu \mathrm{m}$ thick. Their unique shape and structure allow RBCs with particular mechanical characteristics. RBC membranes and the absence of many standard cellular structures (e.g. the have no nucleus) enable them to readily and drastically deform under mechanical forces. RBC deformability is dependent on the properties of the spectrin molecule in the membrane and has significant implications in different diseases related to RBCs.[34; 35] Deformability is an essential property of the RBCs passing through the capillary bed in which vessel dimension are smaller than the diameter of the cells. Deformability also enables transport of new RBCs from bone marrow and the alignment and tank-treading, which reduce bulk viscosity in larger vessels. RBC deformability is largely dependent on the cytoskeleton which in turn is affected by the plasma and other factors.[34] Deformability is also related to the antioxidant function of RBCs. [3] If there is any alteration in oxidation and reduction properties of the RBC, it can result in structural (anatomical) and functional (physiological) changes. It is well proven that ionic imbalance inside RBCs can result from increased intracellular potassium, magnesium and calcium, which can lead to a decrease in intracellular $\mathrm{pH}$ ultimately altering the redox function of RBCs. This structural and functional alteration in RBCs can result in increased aggregability and adherence to the endothelium of the blood vessel. RBC size and deformability are important factors facilitating platelet adherence. [36; 37] With altered RBC deformability, there are significant changes in platelet adherence which further lead to thrombus formation. RBC deformability can be assessed using different techniques such as micropipette aspiration, atomic force microscopy, optical 
tweezers, magnetic twisting cytometry, quantitative phase imaging, ektacytometry, or using microfluidics.[34; 38; 39; 40; 41; 42; 43]

\section{Optical tweezers to asess RBC deformability}

We have used a dual-beam optical tweezers technique [44] to assess deformability of RBCs in patients with DR in comparison to those without DR in patients with type 2 diabetes mellitus (unpublished data). The optical tweezers is constructed on a commercial inverted microscope equipped with a high numerical aperture (NA $=1.3$, oil immersion) $\times 100$ objective lens which both focuses the laser beams to diffractionlimited spots required for optical trapping, and is used for imaging the trapped cells. The trapping beams are derived from a single Nd:YAG laser by splitting and recombining the output using polarisation optics (Figure 2). The position of one trap is fixed, whereas the other can be steered in the transverse plane using computercontrolled galvanometer mirrors in the beam path.

Previous work using optical tweezers to stretch RBCs has used dielectric microbeads attached to the cell as 'handles' for the optical trap to act on. [45; 46] In our work, however, and similar to Rancourt-Grenier et al [47]), we trap the RBC directly. RBCs captured in the optical traps rotate such that they adopt a "side-on" orientation as shown in the inset of Figure 2. All tested RBCs were subject to the same protocol for stretching, achieved by slowly increasing the separation between the beam foci before releasing and allowing the cell to relax back to its natural length. This procedure was repeated up to 30 times on each cell. The cell length during stretching was extracted from digital video recording using custom-written image analysis and edge detection software. A simple deformability index was calculated by measuring the fractional extension of the cell, that is, the change in the cell length normalised by its initial length in each cycle. RBCs from patients with DR were found to have a lower deformability (i.e. when subject to the same stretching protocol the elongation was reduced) compared to control cells from patients without DR and without diabetes. Deformability of the RBCs was found to be inversely correlated with the initial size of RBCs.

\section{RBC Adherence}

Adherence of RBCs to endothelial cells plays a significant role in impediment of blood supply to the tissues through blockages in the microcirculation.[12] [48] The adherence bond between RBC and endothelial cells is reported to be stronger than intercellular bond between RBCs. [12] It hence forms a very potent catalyst for retinal blood vessel occlusion. Illustrative examples can be considered from patients with sickle cell anaemia[48; 49; 50; 51] and falciparum malaria[12] where strong adhesions between abnormal RBCs and endothelial cells result in vaso-occlusive disease. [12] There is increased expression of VCAM-1, E-selectin and ICAM-1 on endothelial cells when they are exposed to sickle RBCs. [49] Increased adhesiveness of RBCs to endothelium is also demonstrated in patients with diabetes 
mellitus; this initiates a cascade of cellular events culminating in protein kinase C activation and also transendothelial migration of monocytes.[52] This diapedesis of activated monocytes hence results in accelerated atherosclerosis, cardiovascular disease and retinopathy in patients with diabetes. [52] There is increased production of cell adhesion molecules (CAM) both in sickle cell disease and diabetes, and hence future treatment could target reduction of CAM expression. [52; 53]

\section{RBC Morphology}

All the circulating blood cells (RBCs, WBCs and platelets) are not only counted but also measured in size and distribution width by automated machines. In the case of RBCs, besides haematocrit, other RBC indices typically measured are the mean corpuscular volume (MCV), mean corpuscular haemoglobin concentration (MCHC), and the RBC distribution width (RDW). [54]

RDW is an automatically measured index of the heterogeneity of RBCs. It is the coefficient of variation of the RBC volume, calculated as the ratio of the standard deviation of the RBC volume and the MCV.[55] Anything more than $14 \%$ is considered as high RDW, which corresponds to $95^{\text {th }}$ percentile of RDW for the reference population in the National Health and Nutrition Examination Survey IIII study.[55] Recently there have been numerous reports speculating about the role of RDW in predicting morbidity and mortality in heart failure patients. [56; 57] According to Tonelli et al.[57] elevated RDW indicates the presence of underlying chronic inflammation which is associated with increased risk of cardiovascular failure. In a separate large cohort study of unselected patients, the authors established the significant correlation of increased RDW and other inflammatory markers in the prognosis of cardiovascular and inflammatory disease.[58] Chronic inflammation has been established as the root cause for atherosclerosis and has also been implicated with ocular diseases like DR, age related macular degeneration (AMD) and vascular occlusion and it will be interesting to investigate RDW in these patients with chronic inflammation affecting the eye.

RDW is so widely and easily available to the clinicians that it can give us insight into some of the diseases at no extra cost to the healthcare.[55] In the large cohort study by Lippi et al. [58] the authors established a significant association between RDW and other inflammatory markers as predictors of CVD or heart failure. A recent study by Vaya et al. [59] documented significant association of increased RDW in active Behcet's disease and as compared to controls.

\section{D reconstruction of $\mathrm{RBC}$ using defocussing microscopy}

Our optical tweezers experiments have demonstrated differences in the mechanical properties of RBCs in the cases of healthy and diabetic patients. We further speculate that differences will also be manifested in other physical properties of $\mathrm{RBCs}$, namely the dimensions such as radius, volume and sphericity index. To measure these quantities we have applied 3D-defocussing microscopy to RBCs, using the algorithm of Roma et al [60] to obtain a 3D reconstruction of the surface of 
a cell from two images at different axial heights. This techniques works by measuring the an additional phase that accrues difference between scattered and non-scattered light from a phase object (such as an RBC) when the microscope is defocussed. For three-dimensional imaging of the cell, contrast intensity measurements of images in two focal positions are made from which information about the phase of the optical electric field can be extracted, and therefore the height profile of the object reconstructed.

In the experiments a selection of five different RBCs is made from every blood sample on which to perform 3D-defocussing microscopy. This selection is made at random from cells that have a healthy appearance, i.e. excluding any that are obviously misshapen or damaged in some way on visual inspection. Once a candidate RBC is selected, three images of the cell at heights $h=-2 \mu \mathrm{m}, 0 \mu \mathrm{m}$ and $2 \mu \mathrm{m}$ relative to the cell center are acquired, see Figures 3(a) and (b). Images are first cropped to a square centered on the cell, then subjected to a shading correction routine in ImageJ that compensates for any fluctuations in the background intensity of the image. The images are binned into a $64 \times 64$ array (performed due to limitations in processing power and memory available, and the exponential scaling of time taken to perform the next processing step as the image size increases) and converted to black and white.

The algorithm for surface reconstruction then determines the height of the refracting surface of the object from contrast measurements in the defocused planes. An example of the reconstructed cell surface obtained by this procedure is shown in Figure 3(c), which has the familiar biconcave disc shape. From the reconstructed cell surface properties such as cell surface area, volume and sphericity can be determined which in future we aim to correlate with deformability measurements.

\subsection{Biochemical properties of $\mathrm{RBC}$ and their role in haemodynamics}

RBCs contain no nucleus or subcellular structure. They are well equipped with nonenzymatic and enzymatic antioxidants to assist RBCs in their scavenging and antioxidant function. RBC membrane is made up of a lipid bilayer and the cytoskeleton. As the RBC membrane needs to be flexible, it is also essential that the lipid domain remains fluidic. Membrane proteins extend from inside the RBC membrane to the outside with the internal and external parts being hydrophilic, whereas the membrane spanning portion between the lipid bilayer is hydrophobic. The membrane spanning portion is classified as integral protein and membrane protein 3 is an important integral protein. The biochemical components of the RBC membrane and also cytoplasm are responsible for the altered mechanical properties of the RBC during disease. The role of RBC biochemical properties in the ocular vascular disorders remains to be elucidated and further research is warranted in understanding the role of RBC biochemical properties in retinal vascular disorders. 


\title{
4. Ocular implications of altered microhaemodynamics - An ophthalmologist perspective
}

\begin{abstract}
4.1 Altered microhaemodynamics in diabetic retinopathy (DR): DR is characterised by vascular and haemodynamic changes which includes retinal blood flow changes, endothelial cell dysfunction, breakdown of the blood retinal barrier, ischaemia and neovascularisation. All the changes in DR are proposed to be induced secondary to oxidative stress and inflammatory process. Hyperglycaemia alters blood components leading to increased production of inflammatory markers, which in turn induce haemodynamic changes in the retinal microvasculature. These changes include increased vascular permeability, vasodilatation and adhesion of inflammatory cells to the blood vessel wall.[61]
\end{abstract}

Haemorheologic factors have long been implicated in the pathogenesis of complications of diabetes. [62]. Compelling evidence suggests that oxidative stress induced by hyperglycemia plays an important role in the development of vascular alterations in the retina. Oxidative stress can be produced by multiple cell types within the retina and retinal vasculature. We have already established the role of leukocytes and leukostasis in DR (unpublished) and there is extensive literature on leukocyte induced changes in DR.

Role of RBC deformability in DR: RBC deformability is necessary for physiological blood flow. It facilitates the blood flow in small vessels, especially capillaries, and hence the oxygenation of tissues and removal of metabolic products. Deformability is primarily affected by cell geometry, cell shape and internal viscosity (determined by hemoglobin concentration and erythrocyte membrane).[63] Changes in membrane lipid-protein interactions along with increased internal viscosity due to glycosylation leads to decreased deformabiity in RBCs in Diabetes.[64] In our experimental set up, we have elucidated the role of RBC deformability in diabetes and DR ( unpublished data).

4.2 Altered blood properties in hypertensive retinopathy: Hypertensive patients, including borderline cases, have been shown to exhibit increased blood viscosity. Letcher et al[65] reported higher plasma viscosity as well as increased haematocrits in hypertensives. In subjects with matched haematocrit values, viscosity remained higher in the hypertensive patients, and the relationship between blood pressure and viscosity was still significant even in borderline cases.[66] The authors suggested that higher fibrinogen levels caused increased plasma viscosity. They also demonstrated a direct correlation between blood pressure and blood viscosity among normotensive and hypertensive subjects. Overall, they suggested that the increased blood viscosity is a consequence of increased haematocrit, plasma viscosity, and RBC aggregation.

4.3 Altered haemodynamics in venous stasis retinopathy and venous occlusion: Glacet-Bernad et al. found elevated RBC aggregation to be an independent and isolated association in patients with central retinal vein occlusion without any conventional risk factors. [62] Wautier et al. demonstrated increased adhesiveness of RBCs from patients with central retinal vein occlusion, as compared to RBCs from 
normal control groups or patients with retinal artery occlusion.[67; 68] The authors concluded that increased adhesion was due to upregulation of phosphatidylserine ( 2.4 fold increase) on the surface of RBCs in patients with vein occlusion.[67]

4.4 Altered haemodynamics in central retinal artery occlusion (CRAO): There is limited clinical data on the haemodynamic changes in CRAO. Kieswetter et al. [69] reported rheologic parameters in patients with acute occlusion of the central retinal artery. They reported increased haematocrit values as well as plasma viscosity. Increased $\mathrm{RBC}$ aggregation with reduced $\mathrm{RBC}$ deformability and the higher plasma fibrinogen levels has been postulated to be the possible hemodynamic pathway in pathophysiology of CRAO or vascular occlusion.

4.5 Altered microhaemodynamics in Behcet's disease: Behcet's disease is a chronic recurrent systemic vasculitis of unknown cause. Owing to the systemic nature of the disease, it affects all organs of the body either clinically or subclinically. There is vasculitis of either vasa vasorum or haemorheological abnormalities in Behcet's disease. The role of RBC deformability in the disease has been investigated, with some studies reporting altered RBC deformability in patients with active Behcet's disease [70] and others no role of RBC deformability in patients with stable or inactive Behcet's disease. [71] Another study has documented that the mean platelet volume does not seem to relate to thrombosis or posterior uveitis in Behcet's disease. [72] However, altered cholesterol metabolism has been shown to be implicated or associated in patients with thrombotic events in Behcet's disease.[73]

4.6 RBC volume and vision loss: Polycythemia vera is a chronic myeloproliferative disorder characterised by increased haematocrit, and hence hyperviscosity, which can potentially lead to vascular occlusion or sagittal sinus thrombosis leading to vision loss.[74] Polycythemia vera should be suspected in patients with elevated haemoglobin or haematocrit levels, splenomegaly, or portal venous thrombosis. There are other secondary causes of increased RBC mass (e.g., heavy smoking, chronic pulmonary disease, renal disease) which must be excluded.[74] There are reported cases of vision loss due to cilioretinal artery occlusion or papilledema as a result of increased RBC mass. Ahn et al. reported a case with monocular vision loss due to polycythemia vera presented with cotton wool spots in the retina and delayed arterial, venous and recirculation time on fluorescein angiogram.[75] After the patient underwent phlebotomy, vision improved dramatically with normalisation of the arterial and venous filling time. This anecdotal case highlights the ischaemic damage of the retina due to elevated blood viscosity.[75]

4.7 Altered microhaemodynamics in other ocular vascular disorders: Systemic lupus erythematosus (SLE) is characterised by activation of complement system and deposition of C3 and C4 complement fragments on plasma cells including RBCs. Ghiran et al studied RBCs from patients with SLE by means of flow cytometry to asses complement fragment deposition on RBC. They concluded that complement activation leads to cytoskeleton changes in RBCs that decreases their deformability and hence their oxygen delivery capacity. [76] 


\section{Fundamental concepts in haemodynamics: A biofluid dynamicists perspective}

\subsection{Defining viscosity}

In the study of haemodynamics, researchers often default to the assumption of Poiseuille flow. The pressure drop, $\Delta p$, required to generate a given flow rate $Q$, for a vessel of diameter $D$ and length $L$ is given by

$R=\frac{\Delta p}{Q}=\frac{128 \mu L}{\pi D^{4}}$

where $\mu$ is the dynamic viscosity of the fluid. This equation for the resistance $R$ of a vessel is defined based on assumptions of steady flow of a Newtonian fluid through a cylindrical rigid tube of infinite length. Clearly, in the context of the vasculature, none of these assumptions are valid. Nonetheless, the equation does yield some useful conclusions, such as the fourth power dependence of resistance on diameter and the proportional increase in resistance with the viscosity $\mu$. The subject of blood viscosity, termed haemorheology, seeks to characterise $\mu$, which has historically, and over-simplistically $[77 ; 78 ; 79]$ been associated with pathology.

In simple terms, the viscosity defines the 'thickness' of a fluid. However, the way in which the term is used in regards to blood flow is very inconsistent. As a result of the cellular components of blood, the viscosity varies as a function of local RBC concentration (haematocrit) and shear rate, which are in turn influenced by RBC deformability and aggregation, the present and upstream vessel dimensions, flow rates and bulk haematocrit. Throughout the vasculature, haematocrit varies on a vessel scale due to plasma skimming [80; 81] and the Fåhraeus effect.[4] Average shear rates in vessels vary by orders of magnitude. Stating that a given blood sample has a 'viscosity' of a specific value is thus insufficient. Furthermore, local distributions of RBCs, viewed in terms of a cell-free layer (CFL)[82; 83], cell-depleted layer (CDL) [84; 85] or a continuous distribution[15; 86; 87; 88] vary significantly. Local distributions of shear rate also vary greatly and depend on geometry and RBC aggregation.

However, it is often not feasible to analyse blood flow at the local level, so bulk values are required. A useful concept in generalising discussions of blood viscosity is to use the 'apparent viscosity', which is the value of $\mu$ which a continuous Newtonian fluid would need in order to give the same value of $R$ observed experimentally for a blood sample. The influence of altered plasma viscosity (or the viscosity of alternative suspending media in in vitro studies), $\mu_{0}$, can be removed by using it to normalise the apparent viscosity to yield the relative apparent viscosity. This term indicates the relative increase in viscosity (or analogously flow resistance) in a given geometry due to the presence of RBCs (predominantly). However, it should be noted that increased plasma viscosity often occurs due to increased plasma protein content, which may play an additional role in RBC aggregation.

In vessels of a certain size, a spatial distribution of viscosity can be described [15], at least in a time-averaged sense. This definition of viscosity refers to the local viscosity of the fluid, if considered as a continuum, but including the spatial distribution of haematocrit and shear rate. As the size of vessels becomes closer to that of the 
RBCs, the continuum definition of 'viscosity' in a time-averaged sense breaks down and individual cells must be considered. This shift in the haemodynamics occurs in vessels around $20 \mu \mathrm{m}[89]$. This discussion is not exhaustive, but serves to introduce the complexity in describing blood 'viscosity'.

5.2 Velocity, shear rate and shear stress

The velocity profile corresponding to Poiseuille flow is given by

$$
u(r)=u_{\max }\left(1-\left(\frac{r}{R}\right)^{k}\right)
$$

Where $r$ is the radial location, $R$ is the vessel radius and $k=2$. In most blood vessels, the velocity profile is blunted, which can be empirically represented by increasing the exponent $k$. The shear rate, which plays a key role in aggregation is given by

$$
\dot{\gamma}(r)=\frac{d u(r)}{d r}=u_{\max } k \frac{r^{k-1}}{R^{k}}
$$

It can be seen from Equation 3 that in the vessel centre $(r=0)$ the shear rate is equal to zero. This has significant implications for RBC aggregation and will be discussed further later. The blunting of the velocity profile observed in blood flow increases the region of low shear rate in the vessel centre. Note that Equation 2 and 3 only apply to very long straight vessel segments, which are rare, and generally haematocrit and velocity profiles tend to be asymmetric.

Wall shear stress (WSS), is an extremely important mechanical force in the context of haemodynamics. Based on Equation 3, the wall shear stress $(r=R)$ can be approximated by

$$
\tau=\mu \dot{\gamma}=\mu \frac{u_{\max } k}{R}
$$

In light of the previous discussion on viscosity, the influence of the term $\mu$ is highly complex and difficult to predict. However, it is commonly oversimplified by either assuming a value based on bulk flow properties of blood at the haematocrit in the large arteries, or by assuming that there are no cells near the vessel wall, and the viscosity is equal to that of the plasma.[90] Additionally, the term $\dot{\gamma}$ can vary greatly due to blunting and asymmetry in velocity profiles. When considering the influence of various parameters on WSS, it is important to keep these matters in consideration.

\section{Highlights}

- There is limited literature on rheological characteristics of the blood in retinal vascular disorders

- Red blood cells deformability and agreegation can play an important role in pathophysiology of retinal vascular disorders

- Experiments to assess red blood cell deformability using Optical tweezers can help us in understanding biomechanical properties of the blood.

- Role of blood viscosity in retinal vascular disorders need to be elucidated. 


\section{Conclusion and Future scope}

There is compelling evidence that detailed analysis of morphological and functional parameters of RBCs can provide insight into the pathophysiology of numerous vascular, degenerative and inflammatory diseases where oxidative stress is one of the associated trigger mechanisms. It can hence be very useful in the long run to apply this basic morphometric and physiologic change in RBC in clinical practice and also in research settings for further mechanistic studies and drug development. As retinal circulation provides us with the unique opportunity to observe blood flow, investigation of RBC flow dynamics in retinal circulation will provide us with important insight into pathophysiology of vascular disorders. The current established tools alongside advance imaging techniques to measure vascular calibres using either static or dynamic vessel analyser should be combined with RBC indices to provide a composite marker of microcirculation in systemic and ocular vascular diseases.

\section{Acknowledgements:}

Dr Rupesh Agrawal has been awarded with Clinician Scientist Career Scheme by National healthcare Group, Singapore and overseas NMRC research training fellowship by Ministry of Health, Singapore. 


\section{References}

[1] S. Berliner, O. Rogowski, S. Aharonov, et al., Erythrocyte adhesiveness/aggregation: a novel biomarker for the detection of low-grade internal inflammation in individuals with atherothrombotic risk factors and proven vascular disease. Am Heart J 149 (2005) 260-7.

[2] O. Rogowski, S. Berliner, D. Zeltser, et al., The erythrosense as a real-time biomarker to reveal the presence of enhanced red blood cell aggregability in atherothrombosis. Am J Ther 12 (2005) 286-92.

[3] M. Minetti, L. Agati, W. Malorni, The microenvironment can shift erythrocytes from a friendly to a harmful behavior: pathogenetic implications for vascular diseases. Cardiovasc Res 75 (2007) 21-8.

[4] H.L. Goldsmith, G.R. Cokelet, P. Gaehtgens, Robin Fahraeus: evolution of his concepts in cardiovascular physiology. Am J Physiol 257 (1989) H1005-15.

[5] A.L. Copley, The Robin Fahraeus memorial lecture. Robin Fahraeus--the scientist and the person. Thromb Res 54 (1989) 521-59.

[6] E. Pretorius, D.B. Kell, Diagnostic morphology: biophysical indicators for iron-driven inflammatory diseases. Integr Biol (Camb) 6 (2014) 486-510.

[7] A.R. Pries, T.W. Secomb, P. Gaehtgens, Biophysical aspects of blood flow in the microvasculature. Cardiovasc Res 32 (1996) 654-67.

[8] A.S. Popel, P.C. Johnson, Microcirculation and Hemorheology. Annu Rev Fluid Mech 37 (2005) 4369.

[9] H.H. Lipowsky, Microvascular rheology and hemodynamics. Microcirculation 12 (2005) 5-15.

[10] O.K. Baskurt, H.J. Meiselman, Blood rheology and hemodynamics. Semin Thromb Hemost 29 (2003) 435-50.

[11] S. Yedgar, A. Koshkaryev, G. Barshtein, The red blood cell in vascular occlusion. Pathophysiol Haemost Thromb 32 (2002) 263-8.

[12] S. Yedgar, D.K. Kaul, G. Barshtein, RBC adhesion to vascular endothelial cells: more potent than RBC aggregation in inducing circulatory disorders. Microcirculation 15 (2008) 581-3.

[13] G. Barshtein, R. Ben-Ami, S. Yedgar, Role of red blood cell flow behavior in hemodynamics and hemostasis. Expert Rev Cardiovasc Ther 5 (2007) 743-52.

[14] W.M. Chan, D.S. Lam, T.Y. Lai, et al., Choroidal vascular remodelling in central serous chorioretinopathy after indocyanine green guided photodynamic therapy with verteporfin: a novel treatment at the primary disease level. Br J Ophthalmol 87 (2003) 1453-8.

[15] J.M. Sherwood, E. Kaliviotis, J. Dusting, S. Balabani, Hematocrit, viscosity and velocity distributions of aggregating and non-aggregating blood in a bifurcating microchannel. Biomech Model Mechanobiol 13 (2014) 259-73.

[16] T. Gori, Endothelium and Hemorheology Handbook of Hemorheology and Hemodynamics, IOS Press, Amsterdam, 2007, pp. 339-350.

[17] Y.C. Fung, Micro- and Macrocirculation, Biomechanics: Motion, Flow, Stress, and Growth., Springer-Verlag New York, New York, 1990, pp. 196-226.

[18] G.G. Tortora, S.R. , Introduction to the human body, John Wiley \& Sons, 2001.

[19] Z. Pecsvarady, T.C. Fisher, C.H. Darwin, et al., Decreased polymorphonuclear leukocyte deformability in NIDDM. Diabetes Care 17 (1994) 57-63.

[20] M.H. Kroll, J.D. Hellums, L.V. McIntire, A.I. Schafer, J.L. Moake, Platelets and shear stress. Blood 88 (1996) 1525-41.

[21] K. Fronek, B.W. Zweifach, Microvascular pressure distribution in skeletal muscle and the effect of vasodilation. Am J Physiol 228 (1975) 791-6.

[22] J. Levick, An introduction to cardiovascular physiology, Hoddler Arnold, 2009.

[23] Y.C. Fung, Biomechanics: Circulation, Springer, 1997.

[24] J. Dupire, M. Socol, A. Viallat, Full dynamics of a red blood cell in shear flow. Proc Natl Acad Sci U S A 109 (2012) 20808-13. 
[25] G.W. Schmid-Schonbein, Biomechanics of microcirculatory blood perfusion. Annu Rev Biomed Eng 1 (1999) 73-102.

[26] R. Wells, H. Schmid-Schonbein, S. Bygdeman, Analysis of viscous deformation of the red cell and its effect upon microvascular flow. Bibl Anat 10 (1969) 92-8.

[27] R. Wells, H. Schmid-Schonbein, Red cell deformation and fluidity of concentrated cell suspensions. J Appl Physiol 27 (1969) 213-7.

[28] J. Dusting, E. Kaliviotis, S. Balabani, M. Yianneskis, Coupled human erythrocyte velocity field and aggregation measurements at physiological haematocrit levels. J Biomech 42 (2009) 143843.

[29] O.K. Baskurt, H.J. Meiselman, RBC aggregation: more important than RBC adhesion to endothelial cells as a determinant of in vivo blood flow in health and disease. Microcirculation 15 (2008) 585-90.

[30] O.K. Baskurt, H.J. Meiselman, Cellular determinants of low-shear blood viscosity. Biorheology 34 (1997) 235-47.

[31] H.J. Meiselman, Red blood cell aggregation: 45 years being curious. Biorheology 46 (2009) 1-19.

[32] O.K. Baskurt, M. Uyuklu, M.R. Hardeman, H.J. Meiselman, Photometric measurements of red blood cell aggregation: light transmission versus light reflectance. J Biomed Opt 14 (2009) 054044.

[33] E. Kaliviotis, M. Yianneskis, On the effect of dynamic flow conditions on blood microstructure investigated with optical shearing microscopy and rheometry. Proc Inst Mech Eng H 221 (2007) 887-97.

[34] N. Mohandas, M.R. Clark, M.S. Jacobs, S.B. Shohet, Analysis of factors regulating erythrocyte deformability. J Clin Invest 66 (1980) 563-73.

[35] N. Mohandas, S.B. Shohet, The role of membrane-associated enzymes in regulation of erythrocyte shape and deformability. Clin Haematol 10 (1981) 223-37.

[36] P.A. Aarts, P.A. Bolhuis, K.S. Sakariassen, R.M. Heethaar, J.J. Sixma, Red blood cell size is important for adherence of blood platelets to artery subendothelium. Blood 62 (1983) 2147.

[37] P.A. Aarts, R.M. Heethaar, J.J. Sixma, Red blood cell deformability influences platelets--vessel wall interaction in flowing blood. Blood 64 (1984) 1228-33.

[38] M. Bessis, N. Mohandas, C. Feo, Automated ektacytometry: a new method of measuring red cell deformability and red cell indices. Blood Cells 6 (1980) 315-27.

[39] M. Bessis, N. Mohandas, [Continuous measurement of cellular deformability, using a diffractometric method]. C R Acad Sci Hebd Seances Acad Sci D 278 (1974) 3263-5.

[40] X. Wang, H. Zhao, F.Y. Zhuang, J.F. Stoltz, Measurement of erythrocyte deformability by two laser diffraction methods. Clin Hemorheol Microcirc 21 (1999) 291-5.

[41] M. Bessis, N. Mohandas, [Red cell deformability, importance of its measurement in clinical medicine]. Schweiz Med Wochenschr 105 (1975) 1568-70.

[42] Y. Park, C.A. Best, K. Badizadegan, et al., Measurement of red blood cell mechanics during morphological changes. Proc Natl Acad Sci U S A 107 (2010) 6731-6.

[43] Y.K. Kim, K; Park, Y, Blood cell-An overview of studies in hematology, Intech, 2012.

[44] P.H. Jones, O.M. Marago, G. Volpe, Optical Tweezers: Principles and Applications, Cambridge University Press, Cambridge, 2015.

[45] S. Henon, G. Lenormand, A. Richert, F. Gallet, A new determination of the shear modulus of the human erythrocyte membrane using optical tweezers. Biophys J 76 (1999) 1145-51.

[46] M. Dao, C.T. Lim, S. Suresh, Mechanics of the human red blood cell deformed by optical tweezers. Journal of the Mechanics and Physics of Solids 51 (2003) 22.

[47] S. Rancourt-Grenier, M.T. Wei, J.J. Bai, et al., Dynamic deformation of red blood cell in dual-trap optical tweezers. Opt Express 18 (2010) 10462-72. 
[48] R.P. Hebbel, R.S. Schwartz, N. Mohandas, The adhesive sickle erythrocyte: cause and consequence of abnormal interactions with endothelium, monocytes/macrophages and model membranes. Clin Haematol 14 (1985) 141-61.

[49] R.P. Hebbel, O. Yamada, C.F. Moldow, et al., Abnormal adherence of sickle erythrocytes to cultured vascular endothelium: possible mechanism for microvascular occlusion in sickle cell disease. J Clin Invest 65 (1980) 154-60.

[50] R.P. Hebbel, Adhesion of sickle red cells to endothelium: myths and future directions. Transfus Clin Biol 15 (2008) 14-8.

[51] R.P. Hebbel, Adhesive interactions of sickle erythrocytes with endothelium. J Clin Invest 100 (1997) S83-6.

[52] V. Rattan, Y. Shen, C. Sultana, D. Kumar, V.K. Kalra, Diabetic RBC-induced oxidant stress leads to transendothelial migration of monocyte-like HL-60 cells. Am J Physiol 273 (1997) E369-75.

[53] R.P. Hebbel, Blockade of adhesion of sickle cells to endothelium by monoclonal antibodies. N Engl J Med 342 (2000) 1910-2.

[54] A. Tefferi, C.A. Hanson, D.J. Inwards, How to interpret and pursue an abnormal complete blood cell count in adults. Mayo Clin Proc 80 (2005) 923-36.

[55] T.C. Evans, D. Jehle, The red blood cell distribution width. J Emerg Med 9 Suppl 1 (1991) 71-4.

[56] G.M. Felker, L.A. Allen, S.J. Pocock, et al., Red cell distribution width as a novel prognostic marker in heart failure: data from the CHARM Program and the Duke Databank. J Am Coll Cardiol 50 (2007) 40-7.

[57] M. Tonelli, F. Sacks, M. Arnold, et al., Relation Between Red Blood Cell Distribution Width and Cardiovascular Event Rate in People With Coronary Disease. Circulation 117 (2008) 163-168.

[58] G. Lippi, G. Targher, M. Montagnana, et al., Relation between red blood cell distribution width and inflammatory biomarkers in a large cohort of unselected outpatients. Arch Pathol Lab Med 133 (2009) 628-32.

[59] A. Vaya, L. Rivera, J. Todoli, et al., Haematological, biochemical and inflammatory parameters in inactive Behcet's disease. Its association with red blood cell distribution width. Clin Hemorheol Microcirc (2013).

[60] P.M.S. Roma, L. Siman, F.T. Amaral, U. Agero, O.N. Mesquita, Total three-dimensional imaging of phase objects using defocusing microscopy: Application to red blood cells. Applied Physics Letters (2014) 104.

[61] W.T. Cade, Diabetes-related microvascular and macrovascular diseases in the physical therapy setting. Phys Ther 88 (2008) 1322-35.

[62] A. Glacet-Bernard, A. Chabanel, F. Lelong, M.M. Samama, G. Coscas, Elevated erythrocyte aggregation in patients with central retinal vein occlusion and without conventional risk factors. Ophthalmology 101 (1994) 1483-7.

[63] P.M. Moriarty, C.A. Gibson, Association between hematological parameters and high-density lipoprotein cholesterol. Curr Opin Cardiol 20 (2005) 318-23.

[64] C. Watala, H. Witas, L. Olszowska, W. Piasecki, The association between erythrocyte internal viscosity, protein non-enzymatic glycosylation and erythrocyte membrane dynamic properties in juvenile diabetes mellitus. Int J Exp Pathol 73 (1992) 655-63.

[65] R.L. Letcher, S. Chien, T.G. Pickering, J.E. Sealey, J.H. Laragh, Direct relationship between blood pressure and blood viscosity in normal and hypertensive subjects. Role of fibrinogen and concentration. Am J Med 70 (1981) 1195-1202.

[66] R.L. Letcher, S. Chien, T.G. Pickering, J.H. Laragh, Elevated blood viscosity in patients with borderline essential hypertension. Hypertension 5 (1983) 757-62.

[67] M.P. Wautier, E. Heron, J. Picot, et al., Red blood cell phosphatidylserine exposure is responsible for increased erythrocyte adhesion to endothelium in central retinal vein occlusion. J Thromb Haemost 9 (2011) 1049-55.

[68] J.L. Wautier, M.P. Wautier, [Molecular basis of red blood cell adhesion to endothelium]. Ann Pharm Fr 69 (2011) 3-6. 
[69] H. Kieswetter, N. Korber, F. Jung, M. Reim, Rheologic findings in patients with acute central retinal artery occlusion. Graefes Arch Clin Exp Ophthalmol 220 (1983) 92-5.

[70] O. Uskudar, A. Erdem, H. Demiroglu, N. Dikmenoglu, Decreased erythrocyte deformability in Behcet's disease. Clin Hemorheol Microcirc 33 (2005) 89-94.

[71] J.M. Ricart, A. Vaya, J. Todoli, et al., Haemorheological alterations in Behcet's disease are not related to a tendency for venous thrombosis. Thromb Res 115 (2005) 399-404.

[72] J.M. Ricart, F. Espana, S. Navarro, et al., Mean platelet volume does not seem to relate to thrombosis or posterior uveitis in Behcet's disease. Clin Hemorheol Microcirc (2012).

[73] J.M. Ricart, A. Vaya, M. Santaolaria, F. Espana, J. Aznar, Dyslipidaemia in Behcet's disease as a thrombotic risk factor. Ann Rheum Dis 65 (2006) 1248-9.

[74] B.J. Stuart, A.J. Viera, Polycythemia vera. Am Fam Physician 69 (2004) 2139-44.

[75] B.Y. Ahn, K.D. Choi, Y.J. Choi, S.Y. Jea, J.E. Lee, Isolated monocular visual loss as an initial manifestation of polycythemia vera. J Neurol Sci 258 (2007) 151-3.

[76] I.C. Ghiran, M.L. Zeidel, S.S. Shevkoplyas, et al., Systemic lupus erythematosus serum deposits C4d on red blood cells, decreases red blood cell membrane deformability, and promotes nitric oxide production. Arthritis Rheum 63 (2011) 503-12.

[77] S. Forconi, T. Gori, The evolution of the meaning of blood hyperviscosity in cardiovascular physiopathology: should we reinterpret Poiseuille? Clin Hemorheol Microcirc 42 (2009) 1-6.

[78] M. Intaglietta, Increased blood viscosity: disease, adaptation or treatment? Clin Hemorheol Microcirc 42 (2009) 305-6.

[79] B.Y. Salazar Vazquez, J. Martini, A. Chavez Negrete, et al., Microvascular benefits of increasing plasma viscosity and maintaining blood viscosity: counterintuitive experimental findings. Biorheology 46 (2009) 167-79.

[80] B.M. Fenton, R.T. Carr, G.R. Cokelet, Nonuniform red cell distribution in 20 to 100 micrometers bifurcations. Microvasc Res 29 (1985) 103-26.

[81] A.R. Pries, K. Ley, M. Claassen, P. Gaehtgens, Red cell distribution at microvascular bifurcations. Microvasc Res 38 (1989) 81-101.

[82] S. Kim, A.S. Popel, M. Intaglietta, P.C. Johnson, Aggregate formation of erythrocytes in postcapillary venules. Am J Physiol Heart Circ Physiol 288 (2005) H584-90.

[83] P.K. Ong, S. Kim, Effect of erythrocyte aggregation on spatiotemporal variations in cell-free layer formation near on arteriolar bifurcation. Microcirculation 20 (2013) 440-53.

[84] W. Reinke, P. Gaehtgens, P.C. Johnson, Blood viscosity in small tubes: effect of shear rate, aggregation, and sedimentation. Am J Physiol 253 (1987) H540-7.

[85] J.M. Sherwood, J. Dusting, E. Kaliviotis, S. Balabani, The effect of red blood cell aggregation on velocity and cell-depleted layer characteristics of blood in a bifurcating microchannel. Biomicrofluidics 6 (2012) 24119.

[86] J.M. Sherwood, D. Holmes, E. Kaliviotis, S. Balabani, Spatial distributions of red blood cells significantly alter local haemodynamics. PLoS One 9 (2014) e100473.

[87] P.A. Aarts, S.A. van den Broek, G.W. Prins, et al., Blood platelets are concentrated near the wall and red blood cells, in the center in flowing blood. Arteriosclerosis 8 (1988) 819-24.

[88] J. Moger, S.J. Matcher, C.P. Winlove, A. Shore, Measuring red blood cell flow dynamics in a glass capillary using Doppler optical coherence tomography and Doppler amplitude optical coherence tomography. J Biomed Opt 9 (2004) 982-94.

[89] G.R. Cokelet, Poiseuille Award Lecture. Viscometric, in vitro and in vivo blood viscosity relationships: how are they related? Biorheology 36 (1999) 343-58.

[90] R.S. Reneman, T. Arts, A.P. Hoeks, Wall shear stress--an important determinant of endothelial cell function and structure--in the arterial system in vivo. Discrepancies with theory. J Vasc Res 43 (2006) 251-69. 
Figures and Legends

Figure 1:Proposed model of Role of RBC in modulation of haemorheolgoical vicious cycle in retinal circulation demonstrating potential role of RBC deformability, RBC adherence and RBC aggregation in retinal vascular disorders. In eyes with circulatory insufficiency, there is possible local hypoxia, acidosis, metabolic debt with or without retinal exudates which can lead to further changes in the retinal tissue. The inflammation in the retinal tissues can lead to production of acute phase reactants and leukocyte activation which further triggers RBC aggregation and reduced deformability. There is also increase adherence of the RBC to the vascular wall and all the changes along with increase in cell depleted layer, can affect blood flow by affecting blood viscosity in the retinal circulation leading to circulatory insufficiency.

Figure 2: Schematic diagram showing set up of dual-beam optical tweezer with sideon image of RBC under dual beam optical tweezer set.

Figure 3: 3-D reconstruction of RBC using defocussing microscopy. (a) Image of the RBC at height $h=0 \mu \mathrm{m}$; (b) Image of the RBC after defocussing the microscope to a height $h=2 \mu \mathrm{m}$; (c) 3D reconstruction of the cell obtained from these images. The colour scale indicates the height of the surface. 
Figure 1: Flow chart of haemorheological factors and retinal vascular disorders

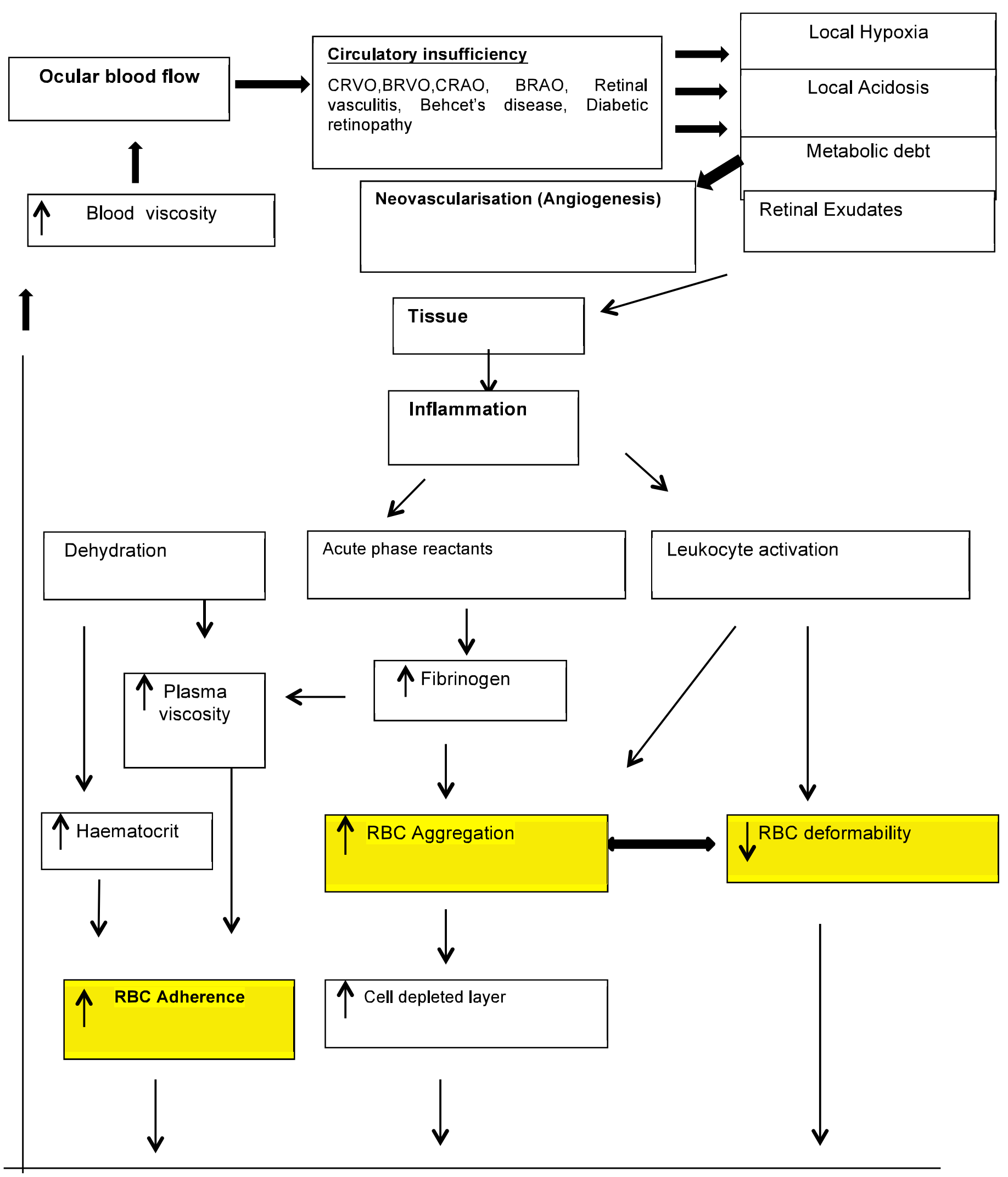



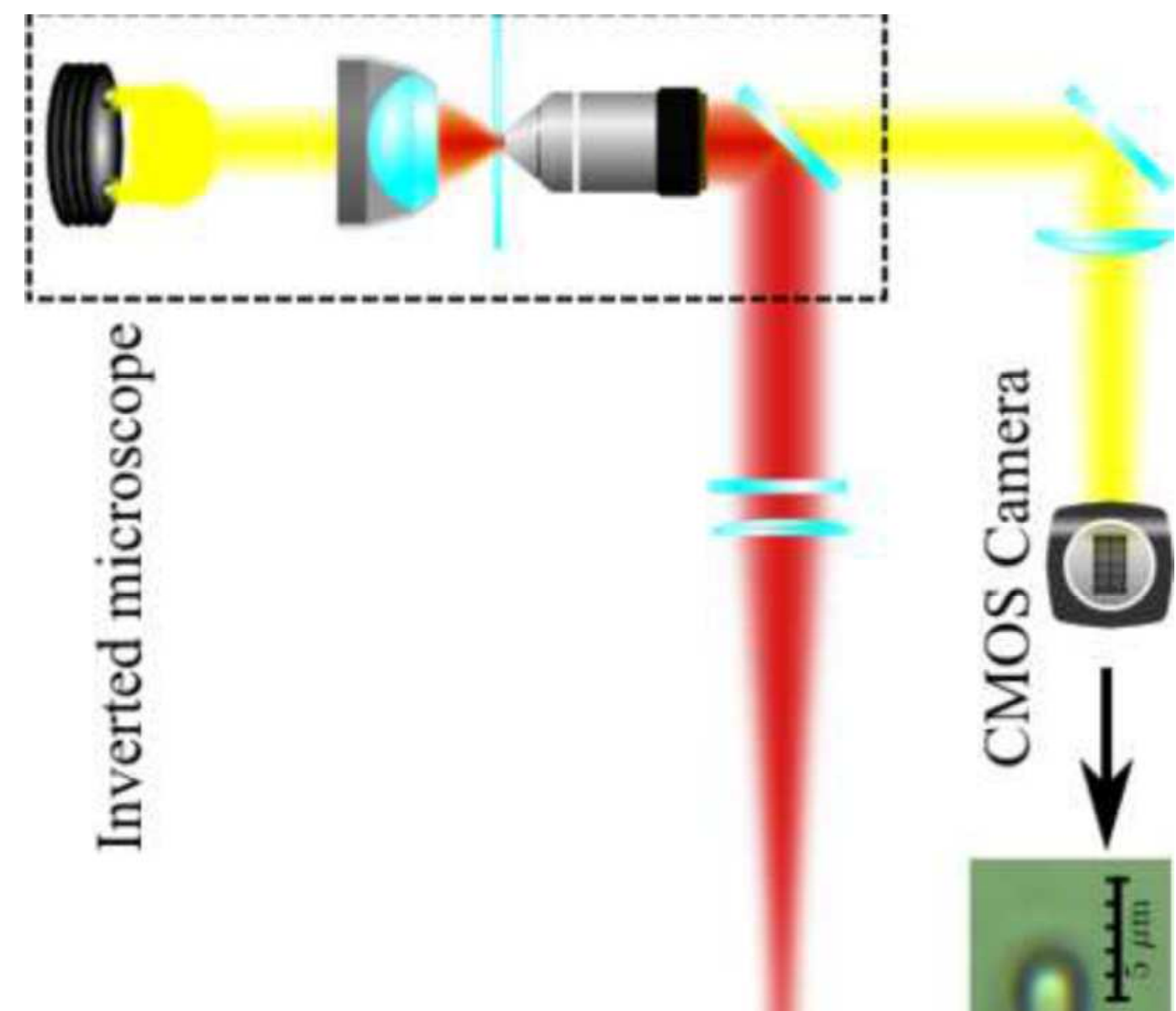

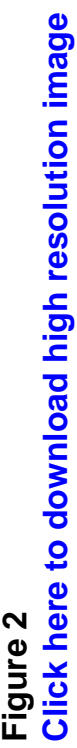

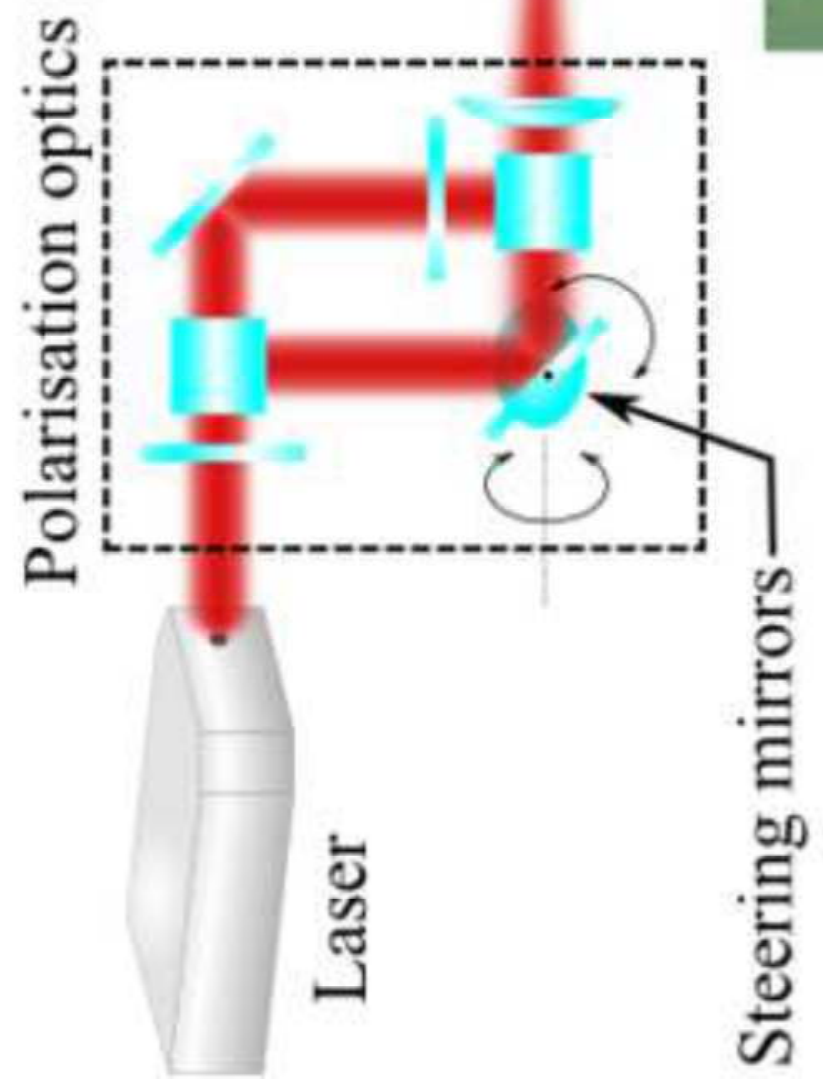



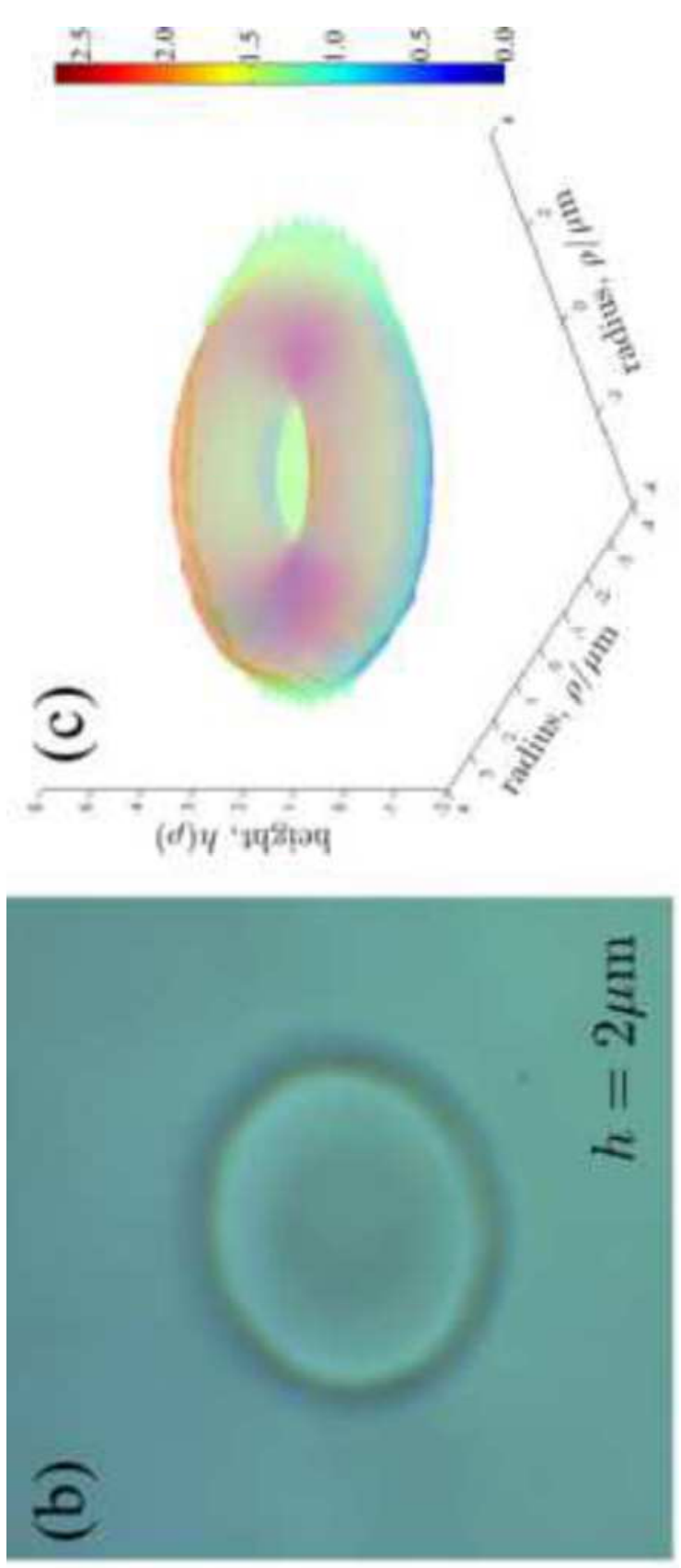

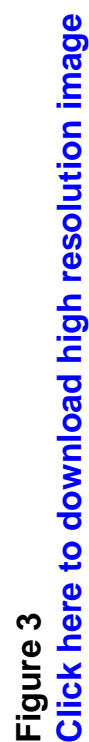

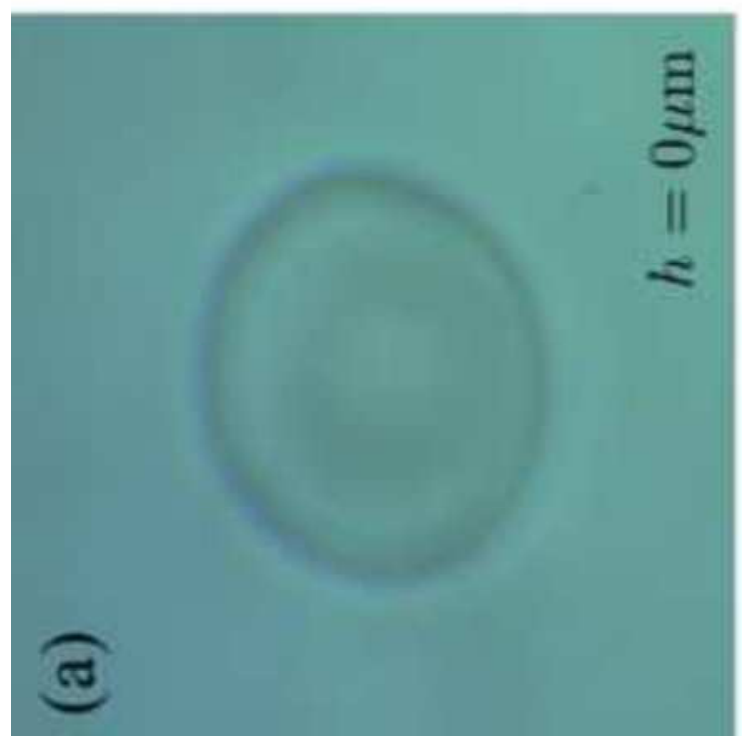

